\title{
原子核强度电势和原子价层电量对元素电负性的标度
}

\author{
余德才 $1^{*}$ 曹文娟 ${ }^{2}$ 余旭东 ${ }^{3}$ \\ ( ${ }^{1}$ 河北工程大学理学院, 河北 甘䣋 056038; 2石家庄经济学院数理学院, 石家庄 050031; \\ 3 复旦大学化学系, 上海 200433)
}

\begin{abstract}
摘要: 根据万有引力势与电势的关系式和系统的对比质电比(单位电量的质量)的物理意义, 定义了系统的强度 电势——单位质量的电势. 研究表明, 元素电负性 $X_{\mathrm{sc}}$ 与原子核在原子边界面上的强度电势 $V_{\mathrm{ic}}$ 和价层电子电量 $q_{\mathrm{cj}}$ 成线性关系. 采用回归分析法, 对周期表中除零族、氢及放射性元素之外的 77 种元素的 Pauling 电负性 $\left(X_{\mathrm{P}}\right)$ 进 行回归分析, 得到了一个无量纲的综合性的电负性计算公式, 相关系数 $(R=0.9844)$ 和回归方程 $F$ 检验的显著性 水平 $(\mathrm{Sig} .=0.0000)$ 表明, $X_{\mathrm{sc}}$ 与 $V_{\mathrm{ic}}$ 和 $q_{\mathrm{cj}}$ 密切相关, 77 种元素的拟合值与 $X_{\mathrm{P}}$ 十分接近, 平均绝对误差仅为 0.084 ; 并预测出较为合理的稀有气体元素的电负性. 用该式可以方便而更准确地计算除氢以外的所有元素的电负性; 同时也给出一条获取离子电负性和基团电负性的新途径.
\end{abstract}

关键词：元素电负性；强度电势；价层电子电量；对比质电比；原子半径 中图分类号: 0641

\section{Scale of Element Electronegativity by Intensive Electrostatic Potential of Atomic Nucleus and Quantity of Electricity of Atomic Valence Shell}

\author{
YU De-Cai ${ }^{1, *} \quad$ CAO Wen-Juan ${ }^{2} \quad$ YU Xu-Dong ${ }^{3}$ \\ ( ${ }^{1}$ College of Science, Hebei University of Engineering, Handan $\quad 056038$, Hebei Province, P. R. China; ${ }^{2}$ School of Mathematics and \\ Science, Shijiazhuang University of Economics, Shijiazhuang 050031, P. R. China; ${ }^{3}$ Department of Chemistry, Fudan University, \\ Shanghai 200433, P. R. China)
}

\begin{abstract}
According to the relationship between gravitational potential and electrostatic potential, as well as considering the physical meaning of the contrastive mass-to-electricity (mass per unit quantity of electricity) a definition of intensive electrostatic potential of the system(the electrostatic potential per unit mass) was proposed. Our research indicates that the electronegativity of elements $\left(X_{\mathrm{Sc}}\right)$ varied with the intensive electrostatic potential of atomic nucleus $V_{\text {ic }}$ and the quantity of electricity of atomic valence shell $q_{\mathrm{cj}}$. A formula for non-dimensional synthetic electronegativity was obtained by multiple regression analysis. The correlation coefficient $(R=0.9844)$ and test of significance (Sig.= $0.0000)$ showed that $X_{\mathrm{Sc}}$ was closely related to $V_{\mathrm{ic}}$ and $q_{\mathrm{c} \text {. }}$. The average absolute error of the 77 determined elemental electronegativities was 0.084 , compared with Pauling electronegativities. The electronegativity of all elements except $\mathrm{H}$ may be calculated conveniently and accurately with our formula, and thus it is a new method for calculating the electronegativity of ions and groups.
\end{abstract}

Key Words: Electronegativities of elements; Intensive electrostatic potential; Electricity of valence shell electrons; Contrastive mass-to-electricity; Atomic radius

元素电负性是化学中常用的基本概念, 它对理 解化学键, 解释化学反应和元素的基本性质等有很

Received: July 4, 2008; Revised: August 12, 2008; Published on Web: November 7, 2008.

"Corresponding author. Email: yudecaigood@163.com; Tel: +86310-8579686.

中国高等教育学会“十一五”教育科学研究规划课题(06AIJ0240031)资助

(C) Editorial office of Acta Physico-Chimica Sinica 
大的帮助. 并且在生物、地质等领域都有着广泛的应 用 ${ }^{[1]}$. 自 1932 年 Pauling 提出元素的电负性概念, 并 将其用作“分子中原子将电子吸引向它自身的能力” 的度量 ${ }^{[2,3]}$ 以来, 人们从不同角度出发, 已经提出了 多种计算元素电负性的标度方法 ${ }^{[4-22]}$, 如基于原子序 数和共价半径的 Sanderson 标度 ${ }^{[4,5]}$, 基于电离能和 电子亲和能的 Mulliken 标度 ${ }^{[0]}$, 基于有效核电荷与 原子半径的 Allred-Rochow 标度 ${ }^{[]]}$和杨立新 ${ }^{[0]}$ 建立的 有效核电荷数的标度等. Allen ${ }^{[9]}$ 将基态自由原子价 壳层电子的平均电子能定义为原子的“光谱电负性”; Parr 等 ${ }^{[10]}$ 利用密度泛函理论(DFT) ${ }^{[11,12]}$ 把电负性和电 子化学势联系起来, 提出了绝对电负性的概念, 对电 负性的研究和发展产生深刻而重要的意义. Nagle ${ }^{[13]}$ 则把原子的极化度、价电子数与原子的电负性关联, 得到的电负性和 Pauling 标度比较接近; 喻典 ${ }^{\left[{ }^{[14]}\right.}$ 用光 谱法测得的原子的价层轨道能量结合共价半径和有 效主量子数, 以静电力为基础计算了价态元素电负 性; 李忠海等 ${ }^{[15}$ 研究了原子价壳层电子量子拓扑指 数与元素电负性的关系, 给出一套电负性数据. 除此 之外其他许多学者也研究了电负性 ${ }^{[16-24]}$. 对于依赖 于特定实验数据的 Pauling 标度 ${ }^{[2]} 、$ Mulliken 标度 ${ }^{[6]}$ 、 Allred-Rochow 标度 ${ }^{[7]}$, 因某些元素的实验数据不全, 只能作出经验推测或造成电负性空缺[5]; 对于以电子 亲和势为基本参数之一的 Mulliken 标度 ${ }^{[6}$ 和 Cao 标 度 ${ }^{[19]}$, 因“元素的电子亲和势数据不全, 测定比较困 难, 准确性比较差”, 难免有 “不全不准”之处, 值得 说明的是, 目前还没有一套完整的为大家所公认的 元素电负性标度 ${ }^{[17]}$. 傅献彩 ${ }^{[25]}$ 指出, 如何采用更好 的方法来表达元素的电负性, 仍有作进一步深人研 究的必要. 本文根据 $\mathrm{Yu}$ 等建立的系统势的模型和 导出的万有引力势与电势的关系 ${ }^{[2,27]}$, 定义了系统的 强度电势, 以原子核的强度电势和原子价层电子电 量为主因素, 通过回归分析得到一个无量纲的、综合 性的元素电负性计算公式.

\section{1 理论基础}

$\mathrm{Yu}$ 等在文献[26,27]中, 建立了统一相互作用的 数理模型一一球形对称系统的势 $(V)$ :

$$
V=k A / r \quad\left(r \geqslant r_{0}\right)
$$

式中 $r$ 为由势点到系统中心的距离, $r_{0}$ 为球形对称 系统的半径, $k$ 为比例常数, $A$ 为系统的 “系统量”. 不同类型的测量工具对应不同“系统量”, 反映系统 势的不同方面, 即不同的“系统量”对应于传统不同
类型的势, 如质量对应于万有引力势, 电量对应于电 势等.引人三个对比参量:

$$
m_{\mathrm{c}}=m / m_{\mathrm{p}}, q_{\mathrm{c}}=q / e_{\mathrm{p}}, r_{\mathrm{c}}=r / r_{\mathrm{p}}
$$

$m_{\mathrm{c}} 、 q_{\mathrm{c}} 、 r_{\mathrm{c}}$ 分别称为对比质量、对比电量、对比距离, $m$ 、 $q$ 分别为系统的质量、电量, $m_{\mathrm{p}} 、 e_{\mathrm{p}} 、 r_{\mathrm{p}}$ 分别为质子质 量、电量、半径. 经推导 ${ }^{[26,27]}$ 得出球形对称系统的万有 引力势与电势的关系:

$$
V_{\mathrm{gc}}= \pm G_{\mathrm{c}} S_{\mathrm{c}} V_{\mathrm{ec}}
$$

式中, $V_{\mathrm{gc}}=-G_{\mathrm{c}} m_{\mathrm{c}} / r_{\mathrm{c}}, V_{\mathrm{ec}}=q_{\mathrm{c}} / r_{\mathrm{c}}\left(r_{\mathrm{c}} \geqslant r_{\mathrm{o}} / r_{\mathrm{p}}\right)$, 分别为对比万 有引力势和对比电势, $G_{\mathrm{c}}$ 为无量纲万有引力常量 ${ }^{[28]}$, $S_{\mathrm{c}}$ 是系统量综合因子, 称为 (对比)质电比:

$S_{\mathrm{c}}=m_{\mathrm{d}} d q_{\mathrm{c}}$

(3)式表明, 球形对称系统的对比万有引力势是对比 电势的 $\pm G_{c} S_{\mathrm{c}}$ 倍(为简便, 以下 “对比”二字将省略). $G_{\mathrm{c}}$ 是两势差异之共性部分一一比例系数; $S_{\mathrm{c}}$ 是系统 差异的综合性因子, 它既包含系统的电性质, 又包含 系统的质量性质, 反映了系统结构的某些综合特征. 所以, 用静态的质量、电量计算出的 $S_{\mathrm{c}}$ 不仅定量地影 响系统的一些静态性质,如系统大小、稳定性等 ${ }^{[27,29]}$, 而且决定着系统在电磁场中的运动状况 ${ }^{[30]}$. 由(3)式 可知, 系统的万有引力势和电势关系依赖于系统的 质电比 $S_{\mathrm{c}}$. 对于相同相态的球对称系统, $S_{\mathrm{c}}$ 越小, 系 统的单位质量的电量越大(单位万有引力势的电势 就越大), 单位质量的电势也越大; 反之亦然. 将 (4) 式变形, 并令 $S_{\mathrm{c}}^{-1}=1 / S_{\mathrm{c}}=q_{\mathrm{c}} / m_{\mathrm{c}}$, 等式两边同除以 $r_{\mathrm{c}}$, 并 令其等于 $V_{\mathrm{ic}}$

$$
V_{\mathrm{ic}}=S_{\mathrm{c}}^{-1} / r_{\mathrm{c}}=\left(q_{\mathrm{d}} / r_{\mathrm{c}}\right) / m_{\mathrm{c}}
$$

与 $V_{\mathrm{ec}}=q_{\mathrm{d}} r_{\mathrm{c}}$ 比较知, $V_{\mathrm{ic}}=S_{\mathrm{c}}^{-1} / r_{\mathrm{c}}$ 正是系统的单位质量 的电势, 定义为强度电势. 其物理意义是系统在 $r_{\mathrm{c}}$ 球面上产生的强度电势, 反映相同质量情况下, 系统 电量对它所吸引带动的其它带电系统一起运动的能 力, 即动态系统吸引带动其它带电系统一起运动的 能力. 它的值越大, 对 $r_{\mathrm{c}}$ 球面上的其它带电系统的 吸引带动作用越强.

\section{2 电负性标度方法}

元素电负性是元素的原子在分子中吸引电子的 能力. 元素的原子与另一种元素的原子以化学键形 成分子是由于价层电子相互作用的结果. 因此, 中性 原子价层电子受原子核引力大小是决定分子中原子 价电子是否容易得失的主要原因, 或者说原子核在 原子边界上产生的势和它在原子边界面上能够吸引 住的价层电子电量的多少是决定分子中成键电子对 
是否偏向该原子的主要因素.

原子核系统都是由核子组成的相同的相态(除 ${ }^{1} \mathrm{H}$ 核属于夸克构成的质子相外), 其集体运动的动 态平衡近似为球形. 因此, 原子核的 $S_{\mathrm{c}}^{-1}$ 是影响原子 核的振动、转动等整体运动的综合性参数, 其数值 不同, 原子核的振动、转动及整体运动程度不同, 所 带动核外电子绕核旋转运动状况不同(原子核的集 体运动速度大于核外电子绕核旋转速度 $\left.{ }^{[31}\right)$. 原子核 的 $S_{\mathrm{c}}^{-1}$ 越大, 原子核的强度电势 $V_{\mathrm{ic}}$ 越大. 若取 $r_{\mathrm{c}}$ 为 (对比) 原子半径 $r_{\mathrm{ca}}$, 则

$$
V_{\mathrm{ic}}=S_{\mathrm{c}}^{-1} / r_{\mathrm{ca}}
$$

该式的意义为: 处于基态的原子, 原子核的单位质 量的电量在原子边界面上任一点处产生的强度电 势, 该值越大, 原子核吸引带动原子边界面上价层电 子旋转运动能力越大, 即动态的原子核带动核外电 子旋转运动的能力越大, 价层电子脱离原子的几率 越小, 元素的电负性就越大. 因此元素的电负性与原 子核在原子边界上的强度电势成正相关. 各原子核 的 $S_{\mathrm{c}}^{-1}$ 和强度电势值列于表 1 . 其中 $S_{\mathrm{c}}^{-1}$ 等于原子核 的电量除以质子电量与原子核的质量除以质子质量 之比, 原子核的质量近似用原子的质量 $m_{\mathrm{a}}{ }^{[32]}$ 减去其 核外电子的质量来计算; 原子半径 $r_{\mathrm{a}}$ 数据取自文献 [33], 零族元素的原子半径数据取自文献[34], $r_{\mathrm{p}}$ 取 $0.8 \times 10^{-15} \mathrm{~m}^{[35]}$. 例如 $\mathrm{O}$ 原子核的 $S_{\mathrm{c}}^{-1}$ 为

$$
\begin{aligned}
& S_{\mathrm{c}}^{-1}=\frac{8 e / e_{\mathrm{p}}}{\left(m_{\mathrm{a}}-8 m_{\mathrm{e}}\right) / m_{\mathrm{p}}}= \\
& \frac{8}{(15.9994-8 \times 0.000548580) / 1.00728}=0.5038
\end{aligned}
$$

这里 $e$ 为元电荷 $、 m_{\mathrm{e}}$ 为电子的质量. $\mathrm{O}$ 原子核在原 子边界上的强度电势为

$$
V_{\mathrm{ic}}=S_{\mathrm{c}}^{-1} / r_{\mathrm{ca}}=0.5038 /\left(0.066 / 0.8 \times 10^{-6}\right)=6.107 \times 10^{-6}
$$

价层电子电量多少也是影响电负性的重要因素. 原 子核的强度电势在原子边界面上能够吸引住的价层 电子电量越多, 价层电子的整体势能就越低(对应于 价层电子都有形成 8 电子等稳定结构的趋势), 吸引 作用越强, 价层电子脱离原子的几率越小, 元素的电 负性就越大. 因此元素的电负性与价层电子电量成 正相关. 假设元素的电负性 $X_{\mathrm{Sc}}$ 与原子核在原子边 界面上的强度电势 $V_{\mathrm{ic}}$ 和价层电子电量 $q_{\mathrm{cj}}$ 的关系满 足数学模型:

$$
\begin{aligned}
& X_{\mathrm{Sc}}=b_{0}+b_{1} V_{\mathrm{ic}}+b_{2} q_{\mathrm{cj}} \\
& q_{\mathrm{cj}}=N e_{\mathrm{d}} e_{\mathrm{p}}=-N
\end{aligned}
$$

$N$ 为价层电子数, 即在数值上价层电子电量等于价 层电子数 $N$ 的负值. 对于主族元素, 价层电子数 $N$ 等于最外层电子数; 对于镧系元素, 价层电子数 $N$ 都取 $n s^{2}$ 上 2 个最外层电子数; 对于过渡元素还要 考虑 $d$ 电子, 由于最外层电子数基本不变, 次外层 $d$ 电子数变化对价电子数有贡献. 可近似用次外层 $d$ 电子的漏屏蔽常数来计量, 依据文献 $[34,36,37]$ 报道 的修正 Slater 电子屏蔽规则, $3 d$ 对 $4 s 、 4 d$ 对 $5 s$ 和 $5 d$ 对 $6 s$ 的屏蔽常数分别为 $0.75 、 0.65$ 和 0.50 . 那么, 第四周期过渡元素每个 $3 d$ 电子的漏屏蔽常数为(1$0.75)=0.25$, 即每增加一个 $3 d$ 电子就相当于增加 0.25 个最外层电子; 第五周期过渡元素每个 $4 d$ 电 子的漏屏蔽常数为 $(1-0.65)=0.35$, 即每增加一个 $4 d$ 电子就相当于增加 0.35 个最外层电子; 第六周期过 渡元素每个 $5 d$ 电子的漏屏蔽常数为 $(1-0.5)=0.5$, 即 每增加一个 $5 d$ 电子就相当于增加 0.5 个最外层电 子. 例如, $\mathrm{Ni}$ 原子电子构型为 $3 d^{8} 4 s^{2}, 8$ 个 $d$ 电子相 当于 $8 \times 0.25=2$ 个最外层电子, 再加上最外层两个 $4 s$ 电子, 共计 4 个最外层电子. 对于 $(n-1) d^{10} n s^{2}$ 电子构 型的元素, 因 $(n-1) d^{10}$ 屏蔽效应显著增大即漏屏蔽效 应显著降低, 所以, $d$ 电子的漏屏蔽常数取同一 $(n-$ 1) $d$ 层中最大的一半较为合适; 对 $(n-1) d^{10} n s^{2} n p^{1}$ 电子 构型的元素, 都存在过渡元素收缩积累的漏屏蔽效 应, 但差异相对较小, 所以, 在最外层 3 个 $n s^{2} n p^{1}$ 电子 基础上, 均加一个电子的漏屏蔽效应, 即共计 4 个最 外层电子较为合适. 各元素的价层电子电量见表 1 .

\section{3 结果与讨论}

以原子核在原子边界上的强度电势 $V_{\mathrm{ic}}$ 和价层 电子电量 $q_{\mathrm{cj}}$ 作为自变量, 以 Pauling 电负性 $X_{\mathrm{P}}{ }^{[33]}$ 为 因变量, 对周期表中除零族、氢及放射性元素之外的 77 种元素的数据进行回归分析, 结果表明, $V_{\mathrm{ic}}$ 和 $q_{\mathrm{cj}}$ 与 Pauling 电负性呈优良的线性相关性, 相关系数 $R=0.9844, F$ 检验的显著性水平 $\mathrm{Sig} .=0.0000$. 将回归 系数 $b_{0}=0.08392 、 b_{1}=0.2803 \times 10^{6}$ 和 $b_{2}=-0.2683$ 代人 (7)式, 得到计算元素电负性的数学公式:

$$
X_{\mathrm{sc}}=0.08392+0.2803 \times 10^{6} V_{\mathrm{ic}}-0.2683 q_{\mathrm{cj}}
$$

用(9)式计算了除氢和放射性元素之外的 82 种元 素的电负性, 结果见表 1 , 同时也列出 Pauling 标度 $\left(X_{\mathrm{P}}\right)^{[33]}$ Allred-Rochow 标度 $\left(X_{\mathrm{A} \mathrm{R}}\right)^{[1]}$ 和 Nagle 标度 $\left(X_{\alpha}\right)^{[13]}$ 以便比较.

由表 1 中数据和图 1 可知, 该电负性标度 $X_{\mathrm{Sc}}$ 与 Pauling 标度非常接近, 上述回归分析的 77 种元

式(7)中 $b_{0} 、 b_{1} 、 b_{2}$ 为回归系数, 式(8)中 $e_{\mathrm{e}}$ 为电子电量, 
素的平均绝对误差仅为 0.084 , 最大的绝对误差也 仅有 0.28. 该平均绝对误差比文献[15]报道的 5 种 代表性标度与 Pauling 标度之间的平均绝对误差最 小的 0.13 (仅比较 65 种元素)还显著地低; 该最大绝 对误差也是最小的, $X_{\mathrm{A}-\mathrm{R}} 、 X_{\alpha} 、 X_{\mathrm{N}}{ }^{[15]}$ 与 $X_{\mathrm{P}}$ 的最大绝对
误差分别为 $0.98 、 1.10 、 0.60$. 这表明 $X_{\mathrm{Sc}}$ 与 Pauling 电负性的一致性显著优于文献[15]报道的几种标 度 ${ }^{[5,7,9,13,15,19]}$, 说明用原子核在原子边界上的强度电 势、价层电子电量标度元素的电负性, 更系统、真实 地描述了分子中原子吸引电子的能力.

表 1 元素电负性 $\boldsymbol{X}_{\mathrm{Sc}}$ 的计算参数及其与 $\boldsymbol{X}_{\mathrm{P}} 、 \boldsymbol{X}_{\alpha}$ 和 $\boldsymbol{X}_{\mathrm{A}-\mathrm{R}}$ 的比较

Table 1 Computational parameters for $X_{\mathrm{Sc}}$ and comparison $X_{\mathrm{Sc}}$ with $X_{\mathrm{P}}, X_{\mathrm{\alpha}}$, and $X_{\mathrm{A} \cdot \mathrm{R}}$

\begin{tabular}{|c|c|c|c|c|c|c|c|c|c|c|c|c|c|c|c|c|c|}
\hline Element & $S_{\mathrm{c}}^{-1}$ & $/ \mathrm{nm}$ & $10^{6} V_{\mathrm{ic}}$ & $-q_{\mathrm{cj}}$ & $X_{\mathrm{sc}}$ & $X_{\mathrm{P}}^{[33]}$ & $X_{\alpha}^{[13]]}$ & $X_{A-R}^{[7]}$ & Element & $S_{\mathrm{c}}^{-1}$ & $r_{a} / \mathrm{nm}$ & $10^{6} V_{\mathrm{ic}}$ & $-q_{\mathrm{cj}}$ & $X_{\mathrm{Sc}}$ & $X_{\mathrm{P}}^{[33]}$ & $X_{\alpha}^{[13]}$ & $X_{A-R}^{[7]}$ \\
\hline $\mathrm{H}$ & 9999 & 0.037 & 21.62 & 1 & $(2.20)$ & 2.20 & 2.27 & 2.20 & $\mathrm{Tc}$ & 0.4421 & 0.136 & 2.600 & 3.75 & 1.82 & 1.90 & 1.30 & 1.36 \\
\hline $\mathrm{He}$ & 5034 & 050 & 8.055 & 2 & 2.88 & & 3.92 & & $\mathrm{Ru}$ & 0.4386 & 0.133 & 2.638 & 4.1 & 1.92 & 2.20 & .35 & 1.42 \\
\hline $\mathrm{Li}$ & 0.4355 & 152 & 2.292 & 1 & 0.99 & 0.98 & 0.94 & 0.97 & $\mathrm{Rh}$ & 0.4406 & 0.134 & 2.630 & 4.45 & 2.02 & 2.28 & 1.39 & 1.45 \\
\hline $\mathrm{Be}$ & 4472 & 0.111 & 3.223 & 2 & 1.52 & 1.57 & 1.55 & 1.47 & $\mathrm{Pd}$ & 1355 & 0.137 & 2.543 & 4.8 & 2.08 & 2.20 & 61 & 1.35 \\
\hline B & 0.4660 & 0.088 & 4.236 & 3 & 2.08 & 2.04 & 2.02 & 2.01 & $\mathrm{Ag}$ & 0.4390 & 0.144 & 2.439 & 4.5 & 1.98 & 1.93 & 1.45 & 1.42 \\
\hline $\mathrm{C}$ & 0.5033 & 0.077 & 5.229 & 4 & 2.62 & 2.55 & 2.55 & 2.50 & $\mathrm{Cd}$ & 0.4302 & 0.149 & 2.310 & 3.4 & 1.64 & 1.69 & 1.45 & 1.46 \\
\hline $\mathrm{N}$ & 0.5035 & 0.070 & 5.755 & 5 & 3.04 & 3.04 & 3.12 & 3.07 & In & 0.4300 & 0.167 & 2.060 & 4 & 1.73 & 1.78 & 1.52 & 1.49 \\
\hline $\mathrm{O}$ & 0.5038 & .066 & 6.107 & 6 & 3.41 & 3.44 & 3.62 & 3.50 & $\mathrm{Sn}$ & 0.4244 & 0.151 & 2.248 & 4 & 1.79 & 1.80 & 1.70 & 1.72 \\
\hline $\mathrm{F}$ & 0.4773 & 0.064 & 5.966 & 7 & 3.63 & 3.90 & 4.23 & 4.10 & $\mathrm{Sb}$ & 0.4220 & 0.145 & 2.328 & 5 & 2.08 & 2.05 & 1.88 & 1.82 \\
\hline $\mathrm{Ne}$ & 0.4993 & 0.131 & 5.472 & 8 & 3.76 & & 4.89 & & $\mathrm{Te}$ & 0.4106 & 0.137 & 2.398 & 6 & 2.37 & 2.10 & 2.08 & 2.01 \\
\hline $\mathrm{Na}$ & 0.4821 & $0.186^{*}$ & 2.073 & 1 & 0.93 & 0.93 & 0.95 & 1.01 & I & 0.4208 & 0.133 & 2.531 & 7 & 2.67 & 2.66 & 2.27 & 2.21 \\
\hline $\mathrm{Mg}$ & 0.4975 & 0.160 & 2.487 & 2 & 1.32 & 1.31 & 1.32 & 1.23 & $\mathrm{Xe}$ & 0.4144 & 0.209 & 2.402 & 8 & 2.90 & & 2.45 & \\
\hline $\mathrm{Al}$ & 0.4855 & 143 & 2.716 & 3 & 1.65 & 1.61 & 1.55 & 1.47 & Cs & 0.4169 & 0.265 & 1.259 & 1 & 0.71 & 0.79 & 0.79 & 0.86 \\
\hline $\mathrm{Si}$ & 0.5022 & 0.117 & 3.434 & 4 & 2.12 & 1.90 & 1.87 & 1.74 & $\mathrm{Ba}$ & 0.4108 & 0.217 & 1.515 & 2 & 1.05 & 0.89 & 0.98 & 0.97 \\
\hline $\mathrm{P}$ & 0.4879 & 0.110 & 3.549 & 5 & 2.42 & 2.19 & 2.22 & 2.06 & $\mathrm{La}$ & 0.4134 & 0.183 & 1.807 & 2 & 1.13 & 1.10 & 1.04 & 1.08 \\
\hline$S$ & 0.5028 & 0.104 & 3.867 & 6 & 2.78 & 2.58 & 2.49 & 2.44 & $\mathrm{Ce}$ & 0.4171 & 0.182 & 1.833 & 2 & 1.13 & 1.12 & 1.05 & 1.06 \\
\hline $\mathrm{Cl}$ & 0.4831 & 0.099 & 3.904 & 7 & 3.06 & 3.16 & 2.82 & 2.83 & Pr & 0.4219 & 0.182 & 1.854 & 2 & 1.14 & 1.13 & 1.06 & 1.07 \\
\hline $\mathrm{Ar}$ & 0.4540 & 0.174 & 3.459 & 8 & 3.20 & & 3.18 & & $\mathrm{Nd}$ & 0.4191 & 0.181 & 1.852 & 2 & 1.14 & 1.14 & 1.03 & 1.07 \\
\hline K & 896 & 232 & 1.688 & 1 & 0.83 & 0.82 & 0.84 & 0.91 & Pn & 0.4239 & 0.183 & 1.853 & 2 & 1.14 & 1.13 & 1.04 & 1.07 \\
\hline $\mathrm{Ca}$ & 0.5028 & 0.197 & 2.042 & 2 & 1.19 & 1.00 & 1.11 & 1.04 & $\mathrm{Sm}$ & 0.4154 & 0.180 & 1.846 & 2 & 1.14 & 1.17 & 1.05 & 1.07 \\
\hline Sc & 1706 & 162 & 2.324 & 2.25 & 1.34 & 1.36 & 1.17 & 1.20 & E & 0.4177 & 0.208 & 1.606 & 2 & 1.07 & 1.20 & 1.06 & 1.01 \\
\hline $\mathrm{Ti}$ & 0.4631 & 0.147 & 2.520 & 2.5 & 1.46 & 1.54 & 1.23 & 1.32 & Gd & 0.4101 & 0.180 & 1.822 & 2 & 1.13 & 1.20 & 1.10 & 1.10 \\
\hline V & 0.4549 & 0.134 & 2.716 & 2.75 & 1.58 & 1.63 & 1.27 & 1.45 & 1 & 0.4121 & 0.177 & 1.862 & 2 & 1.14 & 1.20 & 1.08 & 1.10 \\
\hline $\mathrm{Cr}$ & 0.4651 & 0.128 & 2.907 & 3 & 1.70 & 1.66 & 1.29 & 1.56 & Dy & 4092 & 0.178 & 1.839 & 2 & 1.14 & 1.22 & 1.09 & 1.10 \\
\hline $\mathrm{Mn}$ & 0.4585 & 0.127 & 2.888 & 3.25 & 1.77 & 1.55 & 1.36 & 1.60 & Ho & 0.4093 & 0.176 & 1.860 & 2 & 1.14 & 1.23 & 1.10 & 1.11 \\
\hline $\mathrm{Fe}$ & 4691 & 126 & 2.978 & 3.5 & 1.86 & 1.83 & 1.40 & 1.64 & Er & 0.4096 & 0.176 & 1.862 & 2 & 1.14 & 1.24 & 1.11 & 1.11 \\
\hline Co & 0.4616 & 0.125 & 2.954 & 3.75 & 1.92 & 1.88 & 1.44 & 1.70 & $\mathrm{Tm}$ & 0.4115 & 0.176 & 1.870 & 2 & 1.14 & 1.25 & 1.12 & 1.11 \\
\hline $\mathrm{Ni}$ & 0.4807 & 0.124 & 3.101 & 4 & 2.03 & 1.91 & 1.47 & 1.7 & $\mathrm{Y}$ & 0.4076 & 0.193 & 1.689 & 2 & 1.09 & 1.10 & 1.13 & 1.06 \\
\hline $\mathrm{Cu}$ & 0.4598 & 0.128 & 2.874 & 3.5 & 1.83 & 1.90 & 1.51 & 1.75 & $\mathrm{Lu}$ & 0.4088 & 0.174 & 1.880 & 2.5 & 1.28 & 1.27 & 1.12 & 1.14 \\
\hline $\mathrm{Zn}$ & 0.4622 & 0.134 & 2.760 & 3 & 1.66 & 1.65 & 1.46 & 1.66 & $\mathrm{H}$ & 0.4064 & 0.159 & 2.045 & 3 & 1.46 & 1.3 & 1.20 & 1.23 \\
\hline $\mathrm{Ga}$ & 0.4480 & 0.135 & 2.655 & 4 & 1.90 & 1.81 & 1.56 & 1.82 & $\mathrm{~T}$ & 0.4065 & 0.146 & 2.227 & 3.5 & 1.65 & 1.5 & 1.26 & 1.33 \\
\hline $\mathrm{Ge}$ & 0.4438 & 0.128 & 2.774 & 4 & 1.93 & 2.01 & 1.81 & 2.02 & W & 0.4055 & 0.139 & 2.334 & 4 & 1.81 & 1.7 & 1.31 & 1.40 \\
\hline As & 0.4438 & 0.121 & 2.934 & 5 & 2.25 & 2.18 & 2.11 & 2.20 & $\mathrm{Re}$ & 0.4058 & 0.134 & 2.423 & 4.5 & 1.97 & 1.9 & 1.35 & 1.46 \\
\hline $\mathrm{Se}$ & 0.4338 & 0.117 & 2.966 & 6 & 2.53 & 2.55 & 2.31 & 2.48 & Os & 0.4025 & 0.135 & 2.385 & 5 & 2.09 & 2.2 & 1.39 & 1.52 \\
\hline $\mathrm{Br}$ & 0.4413 & 0.114 & 3.097 & 7 & 2.83 & 2.96 & 2.56 & 2.74 & Ir & 0.4036 & 0.136 & 2.374 & 5.5 & 2.23 & 2.2 & 1.43 & 1.55 \\
\hline $\mathrm{Kr}$ & 0.4328 & 0.189 & 2.910 & 8 & 3.05 & & 2.82 & & $\mathrm{Pt}$ & 0.4028 & 0.139 & 2.318 & 6 & 2.34 & 2.2 & 1.49 & 1.44 \\
\hline $\mathrm{Rb}$ & 0.4362 & 0.248 & 1.407 & 1 & 0.75 & 0.82 & 0.83 & 0.89 & $\mathrm{Au}$ & 0.4041 & 0.144 & 2.245 & 6 & 2.32 & 2.4 & 1.53 & 1.42 \\
\hline $\mathrm{Sr}$ & 0.4370 & 0.215 & 1.626 & 2 & 1.08 & 0.95 & 1.06 & 0.99 & $\mathrm{Hg}$ & 0.4018 & 0.151 & 2.129 & 4 & 1.75 & 1.9 & 1.54 & 1.44 \\
\hline $\mathrm{Y}$ & 0.4420 & 0.180 & 1.964 & 2.35 & 1.27 & 1.22 & 1.11 & 1.10 & $\mathrm{Tl}$ & 0.3993 & 0.170 & 1.879 & 4 & 1.68 & 1.8 & 1.59 & 1.44 \\
\hline $\mathrm{Zr}$ & 0.4418 & 0.160 & 2.209 & 2.7 & 1.43 & 1.33 & 1.17 & 1.22 & $\mathrm{~Pb}$ & 0.3987 & 0.175 & 1.823 & 4 & 1.67 & 1.8 & 1.76 & 1.55 \\
\hline $\mathrm{Nb}$ & 0.4446 & 0.146 & 2.436 & 3.05 & 1.59 & 1.6 & 1.21 & 1.23 & $\mathrm{Bi}$ & 0.4001 & 0.155 & 2.065 & 5 & 2.00 & 1.9 & 1.83 & 1.67 \\
\hline $\mathrm{Mc}$ & 0.4411 & 139 & 539 & 3.4 & .71 & 1.80 & 26 & 30 & & & & & & & & & \\
\hline
\end{tabular}

$r_{\mathrm{a}}$ : atomic radius, $S_{\mathrm{c}}^{-1}$ : the reciprocal of mass-to-electricity of atomic nucleus, $V_{\mathrm{ic}}$ : intensive electrostatic potential of atomic nucleus in atomic boundary, $q_{\mathrm{c} \text { : }}$ : electricity of valence shell electrons, $X_{\mathrm{Sc}}$ : calculated electronegativity of elements in this paper, $X_{\mathrm{p}}$ : Pauling electronegativity, $X_{\mathrm{A}-\mathrm{R}}$ : Allred-Rochow electronegativity, $X_{\alpha}$ : Nagle electronegativity; boldface are the electronegativity of rare gas element calculated by the formulas (9) in this paper. "from Ref.[3,38]. According to the variations of atomic radius in periodic table, or to take all corresponding data of atomic radius to metallic atom,

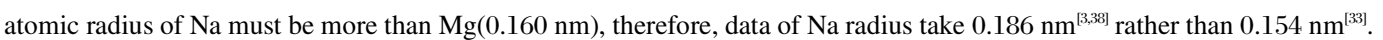




\section{1 主族元素的电负性}

由表 1 及图 1 可见, 主族元素电负性从左向右 随着族次增加电负性依次增加, 从上到下随周期数 的增加逐渐减小, 其中第 IIIA 族出现了交错变化的 情况. 这与 Pauling 标度具有一致的周期性变化规 律. 不同的是 IVA 族中 $\mathrm{Si}$ 和 $\mathrm{Ge}$ 的电负性顺序, 本 标度 $X_{\mathrm{Sc}}(\mathrm{Si})>X_{\mathrm{Sc}}(\mathrm{Ge})$, 与 $X_{\mathrm{P}}$ 相反. 作者认为, 从周期 表中元素位置看, $\mathrm{Si}$ 属典型的非金属, Ge 则属金属, 说明 $\mathrm{Si}$ 的电负性应大于 $\mathrm{Ge}$; 从它们单质及其化合 物的一些性质看, 也说明 $\mathrm{Si}$ 的电负性大于 $\mathrm{Ge}$, 如 $\mathrm{Si}$ 和 $\mathrm{Ge}$ 的氧化物及其水化物, 前者表现弱酸性, 后者 则是两性. 因此, 本标度的顺序是合理的.

关于 $C 、 P 、 S 、 S e 、 T e 、 I 、 H$ 各元素电负性的相对 大小问题, $\mathrm{CS}_{2}$ 和 $\mathrm{CI}_{4}$ 分子中, $\mathrm{C}-\mathrm{S}$ 和 $\mathrm{C}-\mathrm{I}$ 键的电 子云分别偏向 $\mathrm{S}$ 和 $\mathrm{I}$ 原子 ${ }^{[39]}$, 表明 $\mathrm{S}$ 和 $\mathrm{I}$ 的电负性 均大于 $\mathrm{C}$, 而 $X_{\mathrm{A}-\mathrm{R}}{ }^{[7]} 、 X_{\mathrm{M}}{ }^{[6]} 、 X_{\mathrm{Y}}{ }^{[16]} 、 X_{\alpha}{ }^{\left[{ }^{[13]}\right.} 、 X_{\mathrm{Z}}{ }^{[18]} 、 X_{\mathrm{C}}{ }^{[19]}$ 的电 负性标度均为 $\mathrm{C}$ 大于 $\mathrm{S}$ 和 $\mathrm{C}$ 大于 $\mathrm{I}$, 李国胜 ${ }^{[17]}$ 的电 负性标度也为 $\mathrm{C}$ 大于 $\mathrm{S}$, 与键的极性方向相矛盾, 而 本文的标度为 $X_{\mathrm{Sc}}(\mathrm{S})>X_{\mathrm{Sc}}(\mathrm{C})$ 和 $X_{\mathrm{Sc}}(\mathrm{I})>X_{\mathrm{Sc}}(\mathrm{C})$, 与 $\mathrm{C}-\mathrm{S}$ 和 C-I 键的极性方向相一致. P、S、Se、Te、I 的氢化 物中, $\mathrm{P}-\mathrm{H}$ 键偶极矩为 $1.20 \times 10^{30} \mathrm{C} \cdot \mathrm{m}$, 方向指向 磷 ${ }^{[33]}$, 并且磷的氧化数为 -3 , 表明 $\mathrm{P}$ 的电负性大于 $\mathrm{H}$, 而表 1 中列举的 $X_{\mathrm{P}}^{[33]} 、 X_{\mathrm{A}-\mathrm{R}}{ }^{[7]}$ 和 $X_{\alpha}^{[13]} 3$ 种电负性标 度均是 $\mathrm{H}$ 的电负性大于 $\mathrm{P}$, 因此不能对实验结果作 出解释; $\mathrm{H}_{2} \mathrm{~S}, \mathrm{H}_{2} \mathrm{Se}, \mathrm{H}_{2} \mathrm{Te}, \mathrm{HI}$ 均为酸性物质, $\mathrm{H}$ 带部 分正电荷, 在水中上述氢化物均能电离出 $\mathrm{H}^{+}$, 表明 S、Se、Te、I 元素的电负性均大于 $H$, 而表 1 列举的 3 种电负性和文献 $[5 、 6 、 9 、 18 、 19]$ 的电负性标度都是 $\mathrm{H}$ 的电负性大于 $\mathrm{Te}, \mathrm{Mulliken}^{[6]}$ 、李国胜 ${ }^{[17]}$ 、曹晨忠 ${ }^{[19]}$

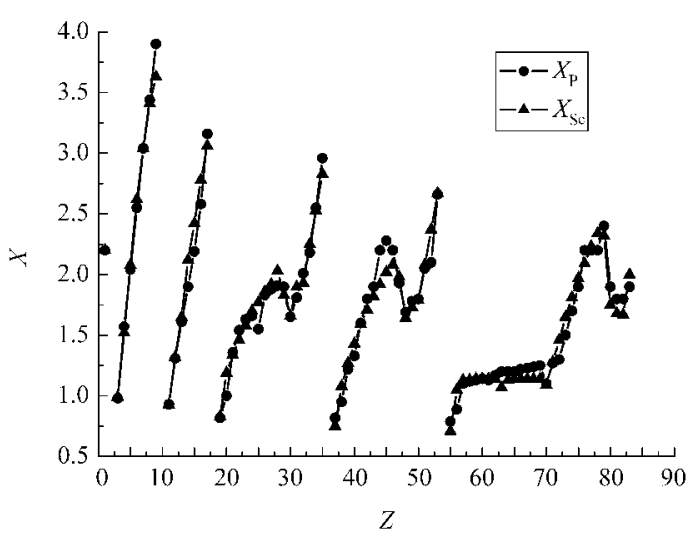

图 1 元素电负性计算值 $\boldsymbol{X}_{\mathrm{Sc}}$ 与 Pauling 标度 $\boldsymbol{X}_{\mathrm{P}}$ 的比较

Fig.1 The comparison of calculated electronegativity $\boldsymbol{X}_{\mathrm{Sc}}$ with Pauling electronegativity $\boldsymbol{X}_{\mathrm{P}}$

$Z$ : atomic number; $X$ : electronegativities of elements
和喻典 ${ }^{[16]}$ 的 4 种电负性标度均为 $\mathrm{H}$ 的电负性大于 $\mathrm{S}$, $\mathrm{Se}, \mathrm{Te}, \mathrm{I}$, 不能有效解释这一实验事实. 本标度中 $\mathrm{P} 、$ $\mathrm{S} 、 \mathrm{Se} 、 \mathrm{Te}, \mathrm{I}$ 的电负性分别为 $2.42 、 2.78 、 2.53 、 2.37$ 、 2.67 , 都大于氢 $\left(X_{\mathrm{Sc}}(\mathrm{H})=X_{\mathrm{P}}=2.20\right.$, 说明见后 $)$, 与实验事 实相符.

\section{2 副族元素的电负性}

对于副族元素. 从左向右电负性依次增加(IIB 族除外); 从上到下随周期数的增加电负性逐渐增 大, 这与 Pauling 标度变化规律基本一致. 尤其是反 映了镧系元素之后第六周期重元素 Hf、Ta、W、Re、 $\mathrm{Os} 、 \mathrm{Ir} 、 \mathrm{Pt} 、 \mathrm{Au} 、 \mathrm{Hg}$ 等核外电子运动的相对论效应影 响, 导致镧系收缩, 使得第六周期重元素较同族第五 周期元素的原子吸引电子的能力相近或较强.

\section{3 稀有气体元素的电负性}

用(9)式还计算了稀有气体元素的电负性(表 1 中黑体字). 由表 1 中数据可知, 同周期稀有气体元 素的电负性最大, 这是原子核在原子边界面上的强 度电势和价层电子电量两因素共同作用的结果, 各 稀有气体元素的 $V_{\mathrm{ic}}$ 不是最大, 但它们吸引带动的 $q_{\mathrm{cj}}$ 都是最多,两因素共同作用使得价层电子的整体 势能最低, 价电子脱离原子的几率最小, 元素的电负 性就最大. 同族中, $\mathrm{Ne}$ 的元素电负性最大, $\mathrm{Ne} 、 \mathrm{Ar}$ 、 $\mathrm{Kr} 、 \mathrm{Xe}$ 的元素电负性依次降低. 这些与元素电负性 周期性递变规律一致, 也与文献[13, 19]报道一致, 且 电负性数值也相近. 许多含 $\mathrm{Xe}-\mathrm{F} 、 \mathrm{Xe}-\mathrm{O} 、 \mathrm{Xe}-\mathrm{N}$ 、 $\mathrm{Kr}-\mathrm{F}$ 键的化合物, 如 $\mathrm{XeF}_{2} 、 \mathrm{XeCl}_{2} 、 \mathrm{KrF}_{2} 、 \mathrm{XeO}_{3}$ 等, 表明 $\mathrm{Xe}$ 和 $\mathrm{Kr}$ 的电负性比 $\mathrm{F}$ 小, 而 $\mathrm{Cl} 、 \mathrm{O} 、 \mathrm{~N}$ 的电负 性大于 $\mathrm{Xe}$, 与本标度一致 ${ }^{[40]}$.

关于 $\mathrm{H}$ 元素的电负性, 本文 1 和 2 节中已说明, 在相同的相态下, 原子核在原子边界上的强度电势 (或 $S_{\mathrm{c}}^{-1}$ ) 对元素电负性的影响是系统性的和定量的. 氢元素的原子核 $\left.{ }^{1} \mathrm{H}\right)$ 是由夸克构成的质子相, 与由 核子(质子和中子)构成的其它原子核的相态不同 ${ }^{[27]}$, 不具有可比性. 由夸克构成的质子相紧密, 电荷相对 较大, 半径相对较小, 质子的强度电势数值相对地 大. 所以公式(9)不适用于计算 $\mathrm{H}$ 的电负性. 考虑到 $X_{\mathrm{Sc}}$ 是用 $X_{\mathrm{P}}$ 回归分析得到的一套数据, 给出了各元 素电负性的相对大小, 反映了各元素电负性的内禀 性和规律性, 并与 $X_{\mathrm{P}}$ 很一致. 所以, 作为一套数据的 一致性特征和要求, 取 $X_{\mathrm{Sc}}(\mathrm{H})=X_{\mathrm{P}}(\mathrm{H})=2.20$ 较合适. 进 一步的研究需要确定两类原子核的相态差异系数.

\section{4 结 论}


在万有引力势与电势的关系基础上, 依据系统 的对比质电比的物理意义, 定义了系统的强度电势. 以原子核在原子(基态)边界上的强度电势和原子价 层电子电量为参数, 建立了一种无量纲的、整体性的 和内禀性的电负性计算公式, 可以很方便地直接计 算除氢以外的所有元素的电负性. 该套数据比已有 的文献值更接近 Pauling 电负性值, 且与 Pauling 电 负性十分相近, 但避免了 Pauling 电负性的经验性; 并且还可以解释已有标度不能解释的某些实验事 实. 由此可见, 本文的标度具有整合性的力学理论基 础和明确的物理意义, 用最基本的数据(质量和电量、 原子半径和价层电子数)更真实地描述了分子中原 子吸引电子的能力. 同时, 为进一步研究离子电负性 和基团电负性提供新的可能途径.

\section{References}

1 Sen, K. D.; Jorgensen, C. K. Electronegativity. New York: Springer-Verlag, 1987: Vol. 66

2 Pauling, L. J. Am. Chem. Soc., 1932, 54: 3570

3 Pauling, L. The nature of the chemical bond. 3rd ed., Ithaca, New York: Cornell University Press, 1960: 126

4 Sanderson, R. T. J. Chem. Educ., 1952, 29: 539

5 Sanderson, R. T. J. Chem. Educ., 1988, 65: 112

6 Mulliken, R. S. J. Chem. Phys., 1934, 2: 782

7 Allred, A. L.; Rochow, E. G. J. lnorg. Nucl. Chem., 1958, 5: 264

8 Yang, L. X. Struct. Chem., 2001, 20(2): 138 [杨立新. 结构化学, 2001, 20(2): 138]

9 Allen, L. C. J. Am. Chem. Soc., 1989, 111: 9003

10 Parr, R. G.; Pearson, R. G. J. Am. Chem. Soc., 1983, 105: 7512

11 Hohenberg, P.; Kohn, W. Phys. Rev., 1964, 136: B864

12 Kohn, W.; Sham, L. J. Phys. Rev., 1965, 140: A1133

13 Nagle, J. K. J. Am. Chem. Soc., 1990, 112: 4741

14 Yu, D. Chin. J. Inorg. Chem., 2005, 21(7): 955 [喻 典. 无机化 学学报, 2005, 21(7): 955]

15 Li, Z. H.; Dai, Y. M.; Wen, S. N.; Nie, C. M.; Zhou, C. Y. Acta Chim. Sin., 2005, 63(14): 1348 [李忠海, 戴益民, 文松年, 聂长 明, 周丛艺. 化学学报, 2005, 63(14): 1348]

16 Yu, D.; Chen, Z. D.; Wang, F.; Li, S. Z. Acta Phys. -Chim. Sin., 2001, 17(1): 15 [喻 典, 陈志达, 王 繁, 李述周. 物理化学学 报, 2001, 17(1): 15]

17 Li, G. S.; Zheng, N. W. Acta Chim. Sin., 1994, 52: 448 [李国胜, 郑能武. 化学学报, 1994, 52: 448]

18 Zhang, Y. H. Inorg. Chem., 1982, 21: 3886

19 Cao, C. Z.; Li, Z. L. Chin. J. Inorg. Chem., 1999, 15(2): 218 [曹 晨忠, 李志良. 无机化学学报, 1999, 15(2): 218]

20 Yu, D. Chemistry, 2005, (7): 555 [喻 典. 化学通报, 2005, (7): 555]

21 Nie, C. M.; Wen, S. N.; Li, Z. H.; Xie, S. P.; Wang, H. Q. Acta Chim. Sin., 2002, 60(2): 207 [聂长明, 文松年, 李忠海, 谢水波,
王宏青. 化学学报, 2002, 60(2): 207]

22 Mullay, J. J. Am. Chem. Soc., 1984, 106: 5842

23 Ghosh, S. K. Int. J. Quantum Chem., 1994, 49: 239

24 Restrepo, G.; Mesa, H.; Llanos, E. J.; Villaveces, J. L. J. Chem. Inf. Comput. Sci., 2004, 44(1): 68

25 Fu, X. C. College chemistry. Beijing: Higher Education Press, 1999: 542, 707 [傅献彩. 大学化学. 北京: 高等教育出版社, 1999: 542, 707]

26 Yu, D. C.; Wang, X. M.; Cao, W. J. Journal of Hebei Normal University(Natural Science Edition, Special Edition), 2006, 30 : 145 [余德才, 王新民, 曹文娟. 河北师范大学学报(自然科学版, 专辑), 2006, 30: 145]

27 Yu, D. C.; Cao, W. J.; Hu, W. P.; Wang, X. M. World Journal of Engineering, 2007, 4(1): 95

28 Yu, D. C.; Cao, W. J.; Wang, X. M. Journal of Hebei University of Engineering(Natural Science Edition), 2007, 24(2): 98 [余德才, 曹文娟, 王新民. 河北工程大学学报(自然版), 2007, 24(2): 98]

29 Yu, D. C.; Cao, W. J.; Yu, X. D.; Li, Y. J. Acta Phys. -Chim. Sin., 2008, 24(5): 880 [余德才, 曹文娟, 余旭东, 李亚娟. 物理化学 学报, 2008, 24(5): 880]

30 Yu, D. C.; Wang, X. M.; Cao, W. J. Science Paper Online, 2007, 4: 4 [余德才, 王新民, 曹文娟. 中国科技论文在线, 2007, 4: 4]

31 Bohr, A.; Mottelson, B. R. Nuclear structure. Vol.1, Second Fascicule. Trans. Group of Nuclear Theory in Department of Physics, Beijing University, Beijing: Science Press, 1976 [原子 核结构. 北京大学物理系原子核理论组译. 第一卷, 第二分册. 北 京: 科学出版社, 1976]

32 Teaching and research section of general chemistry in Zhejiang University. General chemistry. Beijing: Higher Education Press, 2004 [浙江大学普通化学教研室编. 普通化学. 北京: 高等教 育出版社, 2004]

33 Dean, J. A. Lange's handbook of chemistry. 15th ed. Trans. Wei, J. F. Beijing: Science Press, 2003 [兰氏化学手册. 第 15 版. 魏俊 发译. 北京: 科学出版社, 2003]

34 Wen, Y. K. Journal of Chinese University of Science and Technology, 1974, (4): 145 [温元凯. 中国科学技术大学学报, 1974, (4): 145]

35 Zhu, S. L. Atomic physics. Beijing: Higher Education Press, 1999: 401 [褚圣麟. 原子物理学. 北京: 高等教育出版, 1999: 401]

36 Hanson, K. H. Theor. Chim. Acta, 1966, (6): 268

37 Zhou, G. D.; Duan, L. Y. Fundament of structure chemistry. Beijing: Peking Universty Press, 2002 [周公度, 段连运. 结构 化学基础. 北京: 北京大学出版社, 2002]

38 He, F. C.; Zhu, Z. H. Structural chemistry. Beijing: People's Education Press, 1979: 3 [何福城, 朱正和. 结构化学. 北京: 人 民教育出版社, 1979: 3]

39 Xu, G. X. Material structure. Beijing: People's Education Press, 1961: 160 [徐光宪. 物质结构. 北京: 人民教育出版社, 1961: 160]

40 Mai, S. W.; Zhou, G. D.; Li, W. J. Advanced inorganic structural Chemistry. Beijing: Beijing University Press, Hong Kong: Chinese University of Hong Kong Press, 2001: 44 [麦松威, 周公度, 李伟 基. 高等无机结构化学. 北京: 北京大学出版社; 香港: 香港中文 大学出版社, 2001: 44] 